\title{
A partir d'un transparent et d'un article de presse
}

\section{Danielle Chaperon et François Rosset}

\section{(2) OpenEdition}

Journals

Édition électronique

URL : http://journals.openedition.org/edl/819

DOI : $10.4000 /$ edl. 819

ISSN : 2296-5084

\section{Éditeur}

Université de Lausanne

\section{Édition imprimée}

Date de publication : 15 mai 2015

Pagination : 297-300

ISBN : 978-2-940331-45-1

ISSN : 0014-2026

\section{Référence électronique}

Danielle Chaperon et François Rosset, «A partir d'un transparent et d'un article de presse », Études de lettres [En ligne], 1-2 | 2015, mis en ligne le 15 mai 2018, consulté le 20 décembre 2020. URL : http:// journals.openedition.org/edl/819; DOI : https://doi.org/10.4000/edl.819 


\section{À PARTIR D'UN TRANSPARENT ET D'UN ARTICLE DE PRESSE}

«Les échelles du texte». Le titre du colloque et du volume sonne à la fois comme une trouvaille et comme une évidence. Il rend compte à merveille de l'expérience de tout étudiant et de tout enseignant qui a fréquenté, ces trente dernières années, la section de langue et littérature françaises. Je conserve, par exemple, un transparent qui doit dater du début du siècle - nous travaillions alors avec des rétroprojecteurs. Sur cette feuille de rhodoïd, aujourd'hui jaunie et cassante, figure un schéma qui permettait de mettre en valeur, aux yeux des étudiants de première année, la logique du cursus qu'ils allaient entreprendre. Une série d'icônes avaient pour but de figurer le texte littéraire dans tous ses états: des pages, des loupes posées sur ces pages, des livres avec des signets, de petits empilements d'ouvrages, une bibliothèque avec un globe terrestre juché à son sommet. La linguistique et la stylistique étaient censées s'occuper de la lecture rapprochée, l'analyse de texte se pencher sur la page et la situer dans un livre, l'histoire littéraire placer le livre dans une pile puis dans une bibliothèque dont les rayons ne portaient pas uniquement des ouvrages de littérature. Voilà pour la première partie des études (le Bachelor). Dans la seconde partie (le Master), les livres et les pages bougeaient et se métamorphosaient selon qu'on les plaçait sur un rayon ou sur un autre, dans une pile ou dans une autre.

Le cursus n'a pas cessé d'évoluer (malgré les supplications de la Faculté) et résulte actuellement de plus de trente ans de débats, d'expériences et de volonté de perfectionnement. Il n’a jamais été conçu selon une échelle temporelle, pas plus que les chaires n'étaient mises au concours "par siècles" - la classification générique étant elle-même secondaire. Depuis les années 1980, l'accent était mis sur la pluralité 
des approches, la diversité des points de vue et, surtout, la continuité du spectre des échelles. Il y avait certes des seuils, des méthodologies spécifiques et des encyclopédies différentes, mais le passage d'une échelle de plan à une autre s'opérait par «zoom arrière» et "zoom avant", à la manière fluide du cinéma. Chacun des quatre collègues que nous honorons ici régnait officiellement sur l'une des portions du spectre et a contribué par son action à consolider notre structure de formation. Mais, à vrai dire, aucun des quatre ne s'est privé de parcourir toute l'échelle des plans. Leur capacité à étendre leur curiosité à toutes les dimensions des phénomènes linguistiques et littéraires a grandement contribué, en transcendant le partage des tâches, à la cohérence de notre parcours d'études. Avec un tel ADN (encore une échelle), les cursus de français étaient prêts à toutes les hybridations: avec les "mauvais genres", avec l'histoire de l'art, avec la musicologie, avec les linguistes "purs et durs", avec les autres langues. Bref, les échelles ont toujours été partagées et les icônes du transparent jauni, on le comprend, furent surtout un artifice honnête de présentation.

Au milieu de ce jeu trompeur des dimensions, il faut faire droit à une échelle particulière, à l'échelle $1: 1$. L'échelle $1: 1$, bien malin qui saura dire à quoi elle correspond textuellement parlant puisqu'aussi bien toutes les cartes aux échelles dont nous avons parlé peuvent prétendre correspondre au territoire. L'échelle $1: 1$, c'est, dans le domaine qui est le mien (la dramaturgie), l'un des noms de code de la "performance théâtrale" et du «spectacle vivant». Elle établit une sorte de contrat avec le public - un contrat qui garantit la coprésence réelle. Acteur et spectateur sont en effet dans le même espace, dans le même temps, dans le même environnement acoustique, lumineux, atmosphérique et thermique; ils sont ancrés dans le même sol et soumis au même centre de gravité. Or enseigner, c'est être présent, réellement et absolument, ici et maintenant: c'est une performance. Bien sûr, le professeur joue un rôle, le rôle du "prof", d'un prof qui s'affirme à coups de détails vestimentaires, de préciosités de langage, d'audaces rhétoriques. Mais ne nous y trompons pas: le rôle n'est pas celui d'un être de fiction; il est plus proche de l'artiste de cirque que du personnage de roman. Ici, le jongleur lance vraiment les balles et le dompteur est réellement face au lion.

Voyez-vous, quand un professeur comme Jean-Michel, André, Claude, Jean lit un texte - je veux dire devant vous -, il refait in vivo l'expérience de ses relations avec le texte. Je me souviens d'un performeur 
qui se préparait soigneusement avant le spectacle en buvant des litres d'eau teintée de bleu de méthylène; quand il était sur scène, sa sueur et ses larmes coulaient bleues. Je ne dis pas que nos collègues buvaient de l'encre - mais une chose est sûre, ce furent et ce sont d'authentiques performeurs, animés par la langue et par les textes, des virtuoses de l'art vivant qu'est l'enseignement.

\section{Danielle Chaperon \\ Vice-rectrice de l'Université de Lausanne}

Au printemps 2011, le quotidien vaudois 24 Heures consacrait un article à la situation rare vécue par la section de français: quatre de ses professeurs, les plus marquants, les plus connus, les plus identifiés à la section elle-même allaient partir à la retraite pratiquement en même temps. La curée, aurait pu laconiquement constater Jean; paysage à recomposer selon les possibles mots de Claude; changement de registre discursif, dans un idiome plus proche de Jean-Michel; alors qu'André aurait pu, suivant un de ses penchants, déjouer le tragique en l'exposant, pour rappeler en passant que le rejet n'est après tout qu'un procédé de versification.

Chacun aurait donc eu sa manière de qualifier la situation, mais tous, quoiqu'interrogés séparément, ont finalement dit la même chose au journaliste qui espérait peut-être des épanchements d'aigreur, des récriminations ou de sombres prophéties pour l'avenir de cette section décimée. Eh bien non: notre quatuor a tenu sa partie à quatre voix en parfaite harmonie. Chacun a dit la satisfaction que lui avaient donnée ces années à Dorigny et chacun a exprimé plus fortement encore sa pleine confiance quant à l'avenir de la section.

Même attachement engagé à l'institution, même conscience modeste du travail accompli, même foi dans la relève, qu'elle soit ou non incarnée par les élèves qu’on a formés. Et pourtant, il serait difficile de réunir quatre personnalités, quatre caractères, quatre visions du monde, de la littérature et de la culture plus différents.

Celles et ceux qui restent, comme ceux qui les ont rejoints récemment pour les aider à prendre le relais, ont beaucoup de motifs de gratitude. Ils héritent de convictions, de valeurs et de principes qui donnent toute leur 
consistance au métier d'enseigner, à la définition différenciée et mobile d'un champ disciplinaire complexe, à la prospection intellectuelle, à la pratique de la recherche conçue à la fois comme orientation et comme partage. Ils ont appris, beaucoup appris, par la proximité et l'échange, non par imitation imposée. Aujourd'hui, ils sont particulièrement reconnaissants (et un peu fiers aussi, disons-le) de cette confiance qui leur est témoignée par ceux qui, en partant, leur transmettent les rôles majeurs qui sont aussi les plus difficiles et les plus exposés. Mais ils ne pourront pas dire, ces héritiers, qu'on ne leur avait pas montré comment faire pour assumer cela au mieux.

La Faculté des lettres dans son ensemble doit aussi beaucoup à ce brillant carré. Elle aurait mauvaise grâce de ne pas le dire. Bien sûr, il faudrait énumérer les responsabilités endossées tour à tour par chacun d'eux dans des commissions, des conseils, des projets, des programmes, des actions internes et externes et jusqu'au décanat auquel André a donné des impulsions et un style dignes de la plus longue mémoire.

Si l'on parle de prestige et de renommée, nous savons bien, tout le monde sait bien à qui est dû le fait que Lausanne s'est profilée loin à la ronde, comme un lieu de référence en matière de littérature contemporaine, de linguistique textuelle et d'analyse du discours, de littérature de voyage et d'étude culturelle du paysage, d'examen des rapports entre musique et littérature.

Mais ce qu'il faut peut-être relever avant tout, c'est l'engagement assumé par nos quatre collègues, dans des configurations et des circonstances propres à chacun d'eux, pour faire de la Faculté un espace ouvert, où dialoguent les disciplines et interagissent les personnes, en dépit des tendances à la circonscription des champs et au repli qui sont si naturelles à notre milieu quand dominent l'incuriosité ou un sentiment de menace rarement justifié.

Merci donc, quatre fois merci pour tout cela et pour tout ce qu'il faudrait dire encore s'il fallait convoquer une rhétorique circonstancielle ou si la vanité était ce que cultivent en premier lieu nos amis.

François Rosset

Doyen de la Faculté des lettres de l'Université de Lausanne 